\title{
GTSF1 gene may serve as a novel potential diagnostic biomarker for liver cancer
}

\author{
DE-YONG GAO ${ }^{1}$, YUN LING ${ }^{2}$, XIAO-LI LOU ${ }^{1}$, YING-YING WANG $^{1}$ and LIANG-MING LIU ${ }^{1}$ \\ ${ }^{1}$ Department of Infectious Diseases, Songjiang Hospital Affiliated to Shanghai First People's Hospital, \\ Shanghai Jiaotong University, Shanghai 201600; ${ }^{2}$ Department of Infectious Diseases, \\ Shanghai Public Health Clinical Center Affiliated to Fudan University, Shanghai 200083, P.R. China
}

Received June 8, 2016; Accepted October 26, 2017

DOI: $10.3892 / \mathrm{ol} .2017 .7695$

\begin{abstract}
The gametocyte-specific factor 1 (GTSF1) gene participates in DNA methylation and retrotransposon activation in germ cells, particularly during cell proliferation. The present study aimed to assess the level of GTSF1 gene expression in liver cancer tumor tissues, and its role in human hepatoma cell lines in vitro and in a nude mouse model in vivo. GTSF1 gene expression was detected in liver cancer tumor tissues, compared with in healthy controls, via reverse transcription quantitative polymerase chain reaction. An adeno-associated virus vector was used to study tumor stem cell proliferation in vivo. A plasmid expressing GTSF1 was constructed and transfected into various human hepatoma cell lines, in order to analyze the cellular proliferation and apoptosis of liver cancer cells using small interfering (si)RNAs in vitro. In the present study, GTSF1 gene expression was detected in 18/24 (75.0\%) liver cancer tumor tissues from patients with hepatocellular carcinoma (HCC), and elevated GTSF1 expression was identified in the tissue of one of 32 healthy control samples $(3.13 \% ; \mathrm{P}<0.05)$. Notably, the GTSF1 gene was expressed at a higher frequency in AFP-positive HCC samples $(14 / 16,87.50 \%)$ compared with in AFP-negative HCC samples (4/8, 50.0\%; $\mathrm{P}=0.129)$. In addition, there was no statistical significance between GTSF1 expression in non-HBV-infected (71.42\%) and HBV-infected HCC specimens $(76.47 \%)$, as determined by a $\chi^{2}$ test $(\mathrm{P}=0.921)$. It was demonstrated that GTSF1 significantly increased the tumorigenicity of Ad-shNC-transfected (GTSF1-positive)
\end{abstract}

Correspondence to: Dr De-Yong Gao, Department of Infectious Diseases, Songjiang Hospital Affiliated to Shanghai First People's Hospital, Shanghai Jiaotong University, 2901 Caolang Road, Shanghai 201600, P.R. China

E-mail: gaodeyong1970@163.com

Dr Yun Ling, Department of Infectious Diseases, Shanghai Public Health Clinical Center Affiliated to Fudan University, A3 Building, 2901 Caolang Road, Shanghai 200083, P.R. China

E-mail: yun.ling@shaphc.org

Key words: gametocyte specific factor 1 gene, diagnostic biomarker, liver cancer
HepG2 cells in the nude mouse xenograft model, whereas the sizes and weights of the tumors in the GTSF1-negative group were dercreased in comparison with the GTSF1-positive group $(\mathrm{P}<0.05)$. Reduced levels of GTSF1 mRNA, along with fewer and smaller colonies, were identified in two groups of human liver cancer cells treated with with GTSF1-targeting siRNA, when compared with cells without GTSF1 mRNA interference $(\mathrm{P}<0.05)$. In summary, the present study elucidated the GTSF1 mRNA expression pattern in liver cancer, and investigated the potential role of GTSF1 in tumorigenesis. The data suggest an important role for the GTSF1 gene in the molecular etiology of hepatocarcinogenesis, and indicate a potential application of GTSF1 mRNA expression in liver cancer diagnosis and therapy.

\section{Introduction}

Cases of liver cancer may be divided into two categories, based on the primary tumor site: Primary liver cancer (PLC) and metastatic cancer of the liver (liver metastases). Among the various types of PLC, hepatocellular carcinoma (HCC) is the predominant histological form, accounting for the majority of PLC cases (1). An estimated 782,500 incident PLC cases and 745,500 mortalities occurred worldwide in 2012, with China alone reporting $\sim 50 \%$ of the total number of cases and mortalities (2). HCC development is a multi-step process, and $80 \%$ of HCC develops in cirrhotic livers (3). HCCs are clinically heterogeneous and exhibit genetic alterations (4). At present, an early diagnosis of HCC, without pathological verification, is achieved by analyzing serum $\alpha$-fetoprotein (AFP) levels combined with imaging techniques (5). There is an urgent requirement to develop novel molecular tools for assisting early HCC diagnosis, prognosis and treatment stratification. Molecular profiling of gene expression has improved the understanding of the mechanisms of HCC development, allowing the identification of biomarkers for $\mathrm{HCC}$ diagnosis and the stratification of patients with $\mathrm{HCC}$ for prognosis and therapy (6). Takuji Yoshimura et al (7) previously identified that the gametocyte specific factor 1 (GTSF1) gene, a member of the evolutionarily conserved UPF0224 family, is expressed predominantly in male germ cells. It has been suggested that the expression pattern of GTSF1 and its high conservation may serve an important role in germ cell development (8). A 
meta-analysis of gene expression data suggested that, as male GTSF1-knockout mice are sterile owing to a large proportion of dead germ cells, aberrant overexpression of GTSF1 may have a role in the apoptosis resistance of Mycosis Fungoides (MF) (9). In the present study, screening of gene expression in liver cancer samples, all histologically HCC, revealed an association between GTSF1 expression and liver cancer cell proliferation and apoptosis. To the best of our knowledge, there have been no prior studies examining GTSF1 gene expression and its association with liver cancer.

\section{Materials and methods}

Samples. A total of 24 patients with liver cancer and 32 normal liver controls were retrospectively included in the present study at the Songjiang Hospital Affiliated to The Shanghai First People's Hospital (Shanghai Jiaotong University, Shanghai, China) from April 2009 to December 2014. Normal liver tissues were collected from patients undergoing resection of hepatic hemangiomas. Paired liver cancer and adjacent non-tumor liver tissues ( $\geq 1-\mathrm{cm}$ from the tumor edge) were obtained from patients undergoing resection of liver cancer tumors. The clinicopathological data of the patients were collected retrospectively from the Songjiang Hospital Affiliated to The Shanghai First People's hospital databases. No local or systemic treatment had been administered to these patients prior to surgery. The characteristics of patients with and without liver cancer are summarized in Table I.

Samples of the resected tumor specimens and the normal liver controls were stored immediately in liquid nitrogen at $-196^{\circ} \mathrm{C}$ until analysis. Genomic DNA was obtained by digestion with recombinant polymerase chain reaction (PCR)-grade proteinase K (Roche Diagnostics, Basel, Switzerland). Liver samples were pulverized in liquid nitrogen at $-196^{\circ} \mathrm{C}$ for $10 \mathrm{sec}$ prior to incubation in tail-buffer (Roche Diagnostics, Basel, Switzerland). In total, $750 \mu 1$ Tris-EDTA buffer (Invitrogen; Thermo Fisher Scientific, Inc.) with RNase A (20 $\mu \mathrm{g} / \mathrm{ml})$ were added to the digested tails and incubated for $10 \mathrm{~min}$ at room temperature. The samples were subsequently centrifuged at $4^{\circ} \mathrm{C}$ for $10 \mathrm{~min}$ at $10,000 \mathrm{x} \mathrm{g}$. Supernatant containing the genomic DNA $(600 \mu \mathrm{l})$ was transferred into a 2-ml reaction tube and precipitated by adding $60 \mu 13 \mathrm{M} \mathrm{NaAc}$ and 1,200 $\mu 1100 \%$ EtOH. Total RNA was isolated from each of the frozen samples with an RNeasy ${ }^{\circledR}$ Mini kit (Qiagen Benelux B. V., Venlo, The Netherlands), according to the manufacturer's protocol. Ethical approval was granted by the Institutional Review Board of the Songjiang Hospital Affiliated to The Shanghai First People's Hospital, (Shanghai Jiaotong University), and written and informed consent was obtained from all patients.

Serum AFP and hepatitis $B$ virus (HBV) detection. Serum samples were analyzed using an Architect AFP assay (Abbott Pharmaceutical Co., Ltd., Lake Bluff, IL, USA) and results were calculated using the conversion equation as follows: Conversion factor, $0.83 \mathrm{kU} / \mathrm{l}=1 \mu \mathrm{g} / \mathrm{l}$. The results for AFP levels were provided by the Clinical Laboratory of Songjiang Hospital Affiliated to The Shanghai First People's Hospital, Shanghai Jiaotong University, Shanghai, China. In order to detect hepatitis B virus (HBV), serological markers of HBV were quantified using an enzyme immunoassay kit
(Abbott Pharmaceutical Co., Ltd.). HBV DNA was detected with a Cobas TaqMan HBV test version 2.0 (lower limit of detection, $20 \mathrm{IU} / \mathrm{ml}$; Roche Diagnostics).

RNA/DNA extraction and reverse transcription (RT)-quantitative (q)PCR. Total RNA and genomic DNA from human tissue samples cells were obtained from the pathology department (Songjiang Hospital Affiliated to The Shanghai First People's Hospital, Shanghai, China) and were extracted using a GeneJET RNA Purification kit (cat. no. K0731; Thermo Fisher Scientific, Inc.), according to the manufacturer's protocol. Concentrations were quantified using a NanoDrop 1000 (Thermo Fisher Scientific, Inc.). An RT reaction was performed using $1 \mu \mathrm{g}$ total RNA with a High Capacity cDNA Reverse Transcription kit (cat. no. 4368814, Applied Biosystems; Thermo Fisher Scientific, Inc.). The mRNA levels of GTSF1 were determined by qPCR using a SYBR ${ }^{\circledR}$ Green Master Mix kit and an ABI 7500 Real-Time PCR System (Applied Biosystems; Thermo Fisher Scientific, Inc.). The primer sequences were as follows: $\beta$-actin forward, 5'-AAGATGACCCAGATCATG TTTGAG-3' and reverse, 5'-GCAGCTCGTAGCTCTTCT CCAG-3'; and GTSF1 forward, 5'-CACAAGCATCCTGTC TCATGTG-3' and reverse, 5'-CTACACTTCTGGTCTGGG ATTAC-3'. $\beta$-actin was used as an internal control, and relative quantification was conducted using the comparative cycle threshold method (10); the method was used to analyze the relative changes in GTSF1 expression from the qPCR experiments. All PCR reactions were performed under the following conditions: Initial denaturation at $95^{\circ} \mathrm{C}$ for $30 \mathrm{sec}$, followed by annealing for $30 \mathrm{sec}$ at $55^{\circ} \mathrm{C}$ and extension of DNA for $1 \mathrm{~min}$ at $74^{\circ} \mathrm{C}$. These steps were repeated $25-30$ times prior to final extension for $5 \mathrm{~min}$ at $74^{\circ} \mathrm{C}$. The experiments were performed in triplicate.

Vector construction and tumorigenicity assays in nude mice. To construct the GTSF1 expression vector (pcDNA3.0-GTSF1), a gene fragment encompassing the full-length GTSF1 sequence and its 5'- and 3'-flanking regions was amplified and then cloned into the BamHI and EcoRI sites in pcDNA3.0 (Invitrogen; Thermo Fisher Scientific, Inc.). Amplification of DNA fragments corresponding to amino acids 1-167 of the GTSF1 sequence was performed via PCR with primers 5'-CACAAGCATCCTGTCTCATGTG-3' and 5'-GGCAGG GTATCATCTTTCTATTC-3'. The PCR was performed at $95^{\circ} \mathrm{C}$ for $5 \mathrm{~min}$, then $94^{\circ} \mathrm{C}$ for $1 \mathrm{~min}, 65^{\circ} \mathrm{C}$ for $50 \mathrm{sec}$ and $72^{\circ} \mathrm{C}$ for $40 \mathrm{sec}$, for 30 cycles. Extension was performed at $72^{\circ} \mathrm{C}$ for $10 \mathrm{~min}$ and $4^{\circ} \mathrm{C}$ for $10 \mathrm{~min}$, using Phusion PCR Master Mix (Thermo Fisher Scientific, Inc.). GTSF1 DNA was cloned into the pcDNA3.0 expression vector. Digestion products were purified using a NucleoSpin ${ }^{\circledR}$ extract II kit (Macherey-Nagel, Hœrdt, France) and visualized with ethidium bromide, prior to ligation using T4 DNA Ligase (Invitrogen; Thermo Fisher Scientific, Inc.) overnight at $16^{\circ} \mathrm{C}$. The purified plasmid DNA was verified by DNA sequencing using the Sanger sequencing method performed on 3730XL sequencers (Data Collection v3.0 and Sequencing Analysis v5.2; Thermo Fisher Scientific, Inc.). The sequence blast was processed using Blast software (BLAST+, v2.0.0, https://blast.ncbi.nlm .nih.gov/Blast.cgi; Shanghai Sangong Pharmaceutical Co., 
Ltd., Shanghai, China). All experimental procedures were in accordance with the Guide for the Care and Use of Laboratory Animals, Songjiang Hospital Affiliated to The Shanghai First People's Hospital Ethical Guidelines for Animal Experiments. HepG2 cell lines (Shanghai Chinese Academy of Sciences, Shanghai, China) were transfected with pcDNA3.0-GTSF1 [named Ad-shNC (GTSF1-positive)] or pcDNA3.0 empty vector [named Ad-shGTSF1 (GTSF1-negative control)] using Lipofectamine 2000 Transfection Reagent (cat. no. 11668027; Invitrogen; Thermo Fisher Scientific, Inc.), according to the manufacturer's protocols. Transfected pcDNA3.0-GTSF1-transfected cells were incubated at $37^{\circ} \mathrm{C}$ for $1-2$ days and these cells $\left(2 \times 10^{6}\right)$ were suspended in $100 \mu 1$ PBS prior to being injected subcutaneously into either side of the posterior flank of each 5-6-week-old male $\mathrm{BALB} / \mathrm{c}$ athymic nude mouse. A total of 12 mice were divided into two groups (Shanghai Chinese Academy of Sciences, Shanghai, China). A total of 6 mice were injected with Ad-shNC (GTSF1-positive)-transfected HepG2 cells (total of $2 \times 10^{6}$ targeted cells) and the other groupwasinjected with Ad-shGTSF1 (GTSF1-negative control)-transfected HepG2 cells. The mice were housed in polypropylene cages under standard experimental conditions $\left(20-22^{\circ} \mathrm{C}, 55 \%\right.$ humidity, food and water ad libitum, $12 \mathrm{~h}$ light/dark cycle) and checked at least once a week until tumors became palpable. Mice were sacrificed using 20-30\% $\mathrm{CO}_{2}$ gas flow. Tumor sizes did not exceed $20 \mathrm{~mm}(2.0 \mathrm{~cm})$ in any direction in an adult mouse and tumor growth was observed for $>8$ weeks (pre-determined end-point). The tumor dimensions were measured every 3 days using a digital caliper and the tumor volume was calculated using the following formula: $V=\pi / 6 \times$ (larger diameter) $\mathrm{x}$ (smaller diameter) ${ }^{2}$.

RNA oligoribonucleotides and cell transfections. The small interfering RNA (siRNA) targeting human GTSF1 mRNA (NM_144594.2; https://www.ncbi.nlm .nih.gov/nuccore/NM_144594.2; accessed October 27, 2017; NC_000081.6; https://www.ncbi.nlm.nih .gov/nuccore/NC_000081.6; accessed November 10, 2017) were designated as siRNA1 (5'-UUCUCCGAACGU GUCACGUdTdT-3') and siRNA2 (5'-ACGUGACAC GUUCGGAGAAdTdT-3'). The two siRNAs against GTSF1 were designed using the Whitehead Institute Web Server (http://jura.wi.mit.edu/bioc/siRNAext/) and were chemically synthesized by Shanghai GenePharma Co., Ltd. (Shanghai, China) to target GTSF1 mRNA. The control RNA duplex (NC) for siRNA1 and siRNA 2 was non-homologous to any human genome sequences. For the in vivo tumorigenicity assay, all pyrimidine nucleotides in the NC or siRNA1 and siRNA2 duplex were substituted with their 2-O-methyl analogs to improve RNA stability. The anti-GTSF1 mRNA with the following sequences: siRNA1 (228-250) forward, 5'-GGCUACUUGUCCCUUCAAUDTDT-3' and reverse, 5'-AUUGAAGGGACAAGUAGCCDTDT-3'; and siRNA2 (478-500) forward, 5'-CCUGCGAGCAACAUAGUUAdT dT-3' and reverse, 5'-UAACUAUGUUGCUGCAGGdTdT-3', were 2-O-methyl-modified oligoribonucleotides designed as an inhibitor of GTSF1 mRNA. All RNA oligoribonucleotides were purchased from GenePharma (Shanghai GenePharma Co., Ltd., Shanghai, China). Reverse transfection of RNA
Table I. Patients characteristics with or without HCC.

\begin{tabular}{|c|c|c|c|}
\hline $\begin{array}{l}\text { Clinical } \\
\text { parameters }\end{array}$ & $\begin{array}{c}\text { Liver } \\
\text { cancer }(n=24)\end{array}$ & $\begin{array}{c}\text { Normal } \\
\text { controls }(n=32)\end{array}$ & P-value \\
\hline $\begin{array}{l}\text { Age (years), mean } \\
\pm \mathrm{SD}\end{array}$ & $63.1 \pm 9.9$ & $51.6 \pm 12.7$ & 0.654 \\
\hline \multicolumn{4}{|l|}{$\operatorname{Sex}(\%)$} \\
\hline Male & $18(75.0)$ & $21(65.6)$ & 0.642 \\
\hline Female & $6(25.0)$ & $11(34.4)$ & 0.591 \\
\hline \multicolumn{4}{|l|}{ AFP level (\%) } \\
\hline Normal & $8(33.3)$ & $30(93.8)$ & $0.001^{\mathrm{a}}$ \\
\hline Abnormal & $16(66.7)$ & $2(6.2)$ & $0.001^{\mathrm{a}}$ \\
\hline \multicolumn{4}{|l|}{ HBV infection (\%) } \\
\hline Positive & $17(70.8)$ & $3(9.4)$ & $0.001^{\mathrm{a}}$ \\
\hline Negative & $7(29.2)$ & $29(90.6)$ & $0.001^{\mathrm{a}}$ \\
\hline
\end{tabular}

${ }^{\mathrm{a}} \mathrm{P}<0.01$. Statistical differences between the HCC and healthy control groups were evaluated using the $\chi^{2}$ test for qualitative variables and the unpaired Student's t-test for quantitative variables. HCC, hepatocellular carcinoma; AFP, $\alpha$-fetoprotein; HBV, hepatitis B virus; SD, standard deviation.

oligoribonucleotide(s) was performed using Lipofectamine RNAiMAX $^{\circledR}$ (Invitrogen; Thermo Fisher Scientific, Inc.), according to the manufacturer's protocol. The transfection efficiency, examined by fluorescein amidite-conjugated siRNA and fluorescence-activated cell sorting analysis, was $\sim 75 \%$ in PLC/PRF/5 and Huh-7 cell lines (Shanghai Chinese Academy of Sciences). A total of $50 \mathrm{nmol} / 1$ RNA duplex and $200 \mathrm{nmol} / \mathrm{l} \mathrm{miRNA}$ inhibitor were used for each transfection. The above siRNAs were transfected into HCC PLC/PRF/5 and Huh-7 cell lines. In total, $3 \times 10^{3}$ cells from each cell line were seeded onto 96-well plates. The siRNA oligomer was diluted in $50 \mu$ l Opti-MEM ${ }^{\circledR}$ I Reduced Serum medium without serum (with a final RNA concentration of $40 \mathrm{nM}$ ), prior to being diluted $1 \mu \mathrm{l}$ in $50 \mu \mathrm{l}$ OptiMEM $^{\circledR}$ I Reduced Serum medium, mixed gently and incubated for $5 \mathrm{~min}$ at room temperature. Following incubation, the diluted oligomer was combined with the diluted Lipofectamine ${ }^{\mathrm{TM}}$ 2000, mixed gently and incubate for a further $20 \mathrm{~min}$ at room temperature. The cells were then incubated at $37^{\circ} \mathrm{C}$ in a $5 \% \mathrm{CO}_{2}$ incubator for 24-96 h until subsequent experimentation. Subsequently, $24 \mathrm{~h}$ following the siRNA transfection of HCC PLC/PRF/5 and Huh-7 cells, the cells were transfected with $200 \mathrm{ng}$ plasmid in a 24 -well plate using Lipofectamine $2000^{\circledR}$ (Invitrogen; Thermo Fisher Scientific, Inc.).

Cell growth and colony formation assay. Cell growth was determined by using an MTS assay (Promega Corporation, Madison, WI, USA). Briefly, siRNA-transfected HCC PLC/PRF/5 and Huh-7 cells transfected with either empty vectors or 3xFlag-tagged Gls2 expressing vectors (Sigma-Aldrich; Merck KGaA, Darmstadt, Germany) were cultured at $37^{\circ} \mathrm{C}$ in a 96-well plate in complete Dulbecco's modified Eagle's medium (DMEM; Gibco; Thermo Fisher Scientific, Inc.) at $37^{\circ} \mathrm{C}$ for 2 weeks. This MTS Cell Proliferation 
assay is based on the reduction of MTS tetrazolium compound by viable cells to generate a colored formazan product that is soluble in cell culture media. The quantity of formazan dye produced by viable cells can be quantified and measured at $450 \mathrm{~nm}$ absorbance after $1 \mathrm{~h}$ of incubation at $37^{\circ} \mathrm{C}$ with CellTiter $96^{\circledR}$ Aqueous One Solution Reagent (Promega Corporation, Madison, WI, USA, according to the manufacturer's protocol. Following $24 \mathrm{~h}$ of transfection at $37^{\circ} \mathrm{C}, 3 \times 10^{3}$ siRNA-transfected HCC PLC/PRF/5 and Huh-7 cells were placed in a fresh 6-well plate and maintained in DMEM containing 10\% fetal bovine serum (Gibco; Thermo Fisher Scientific, Inc.) for 2 weeks. Colonies were fixed at room temperature for $30 \mathrm{~min}$ in $1 \%$ methanol and stained at room temperature with $0.1 \%$ crystal violet in $20 \%$ methanol for $15 \mathrm{~min}$.

Statistical analysis. The numerical data are expressed as the mean \pm standard deviation. Differences in proportion were analyzed by the $\chi^{2}$ test or an unpaired Student's t-test and one-way analysis of variance (ANOVA) with a Student-Newman-Keuls (S-N-K) post hoc test, as required. The odds ratios and $95 \%$ confidence intervals were calculated along with Fisher's exact P-values, where appropriate. All calculations were performed with SPSS software version 19.0 (SPSS Inc., Chicago, IL, USA). All experiments were repeated at least three separate times. $\mathrm{P}<0.05$ was considered to indicate a statistically significant difference.

\section{Results}

GTSF1 mRNA expression in human liver cancer tissues and its association with AFP levels. The GTSF1 mRNA expression profiles of 32 normal liver and 24 primary liver cancer samples, which were histologically HCC, were analyzed. When comparing the 24 samples from patients with liver cancer and their adjacent tissues, 22 exhibited significant differential expression of GTSF1 mRNA in liver cancer tissues (Fig. 1). Only 1/32 healthy controls exhibited GTSF1 expression, with a complete lack of expression being observed in the other 31 healthy subjects and in the adjacent non-cancerous liver tissues of the 24 patients with liver cancer. A higher frequency of GTSF1 mRNA expression was observed in the samples from patients with HCC, compared with the healthy control samples, as determined using an unpaired Student's t-test $(\mathrm{P}<0.05)$. To examine the association between AFP levels and GTSF1 mRNA expression, the $\chi^{2}$ test was used to analyze the potential clinical implications of GTSF1 mRNA expression. A total of 4/8 (50\%) samples with negative AFP levels exhibited GTSF1 mRNA expression, and 14/16 (87.5\%) samples with positive AFP levels exhibited GTSF1 mRNA expression (Fig. 1A and B). Although the ratio analysis of the AFP-positive liver cancer samples (87.5\%) was higher compared with the AFP-negative liver cancer samples (50\%), no statistical significance between these two groups was observed using a $\chi^{2}$ test $(\mathrm{P}=0.129)$. In addition, GTSF1 expression was also compared in Hepatitis B virus (HBV)-infected $\mathrm{HCC}$ samples and non-HBV-infected patient specimens to examine the association between HBV infection and GTSF1 mRNA expression. A total of 5/7 (71.42\%) samples that were HBV-negative exhibited GTSF1 mRNA expression, and 13/17 (76.47\%) samples that were HBV-positive exhibited GTSF1

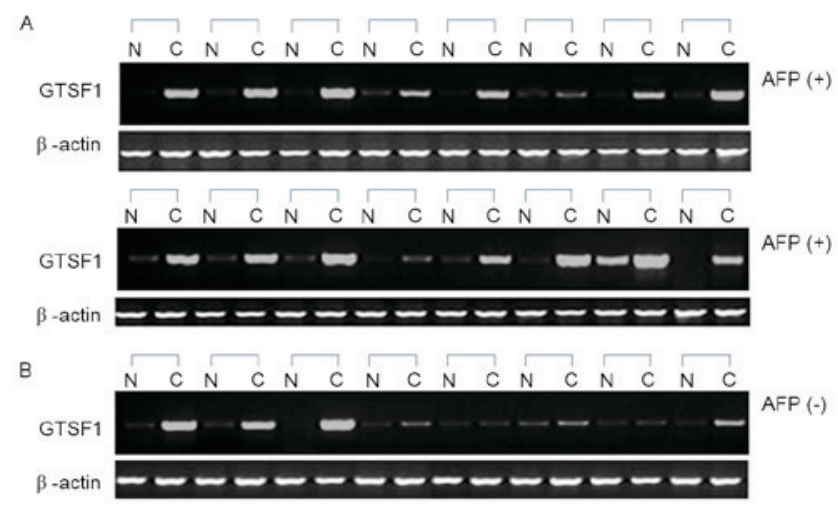

Figure 1. Expression levels of GTSF1 mRNA in 24 paired liver cancer and adjacent non-tumor tissues, evaluated via reverse transcription quantitative polymerase chain reaction. (A) Analysis of GTSF1 mRNA expression levels in HCC tissue samples from 16 AFP-positive patients. (B) Analysis of GTSF1 mRNA expression levels from 8 patients with AFP-negative liver cancer tissues. $\beta$-actin served as the internal control. $\mathrm{N}$, adjacent noncancerous liver; C, HCC tissue; AFP, $\alpha$-fetoprotein; GTSF1, gametocyte-specific factor 1 gene.

mRNA expression. No statistical significance between these two groups was observed, using a $\chi^{2}$ test $(\mathrm{P}=0.921)$.

Vector construction and GTSF1 mRNA raises tumorigenicity in vivo. The significant overexpression of GTSF1 in liver cancer samples prompted an examination of the potential biological significance of GTSF1 in tumorigenesis. In order to confirm the function of GTSF1 mRNA in liver cancer tumorigenicity, an Ad-shNC vector, which was GTSF1-expressing (positive), was constructed using GTSF1 mRNA gene sequencing. A negative control without the GTSF1 vector sequence, termed Ad-shGTSF1 (GTSF1-negative control), was also constructed. As an initial step, the capacity of colony formation was evaluated in the HepG2 cells, which were transfected with the Ad-shNC vector (GTSF1-positive) or the Ad-shGTSF1 (GTSF1-negative) control duplex. Ad-shNC vector- and Ad-shGTSF1-transfected HepG2 cells $\left(5 \times 10^{6}\right.$ in $\left.100 \mu \mathrm{l}\right)$ were injected subcutaneously into 6 nude mice. As demonstrated in Fig. 2A, the tumor became visible at 10-30 days in the mice injected with Ad-shNCtransfected (GTSF1-positive) HepG2 cells, and grew from 10-700 $\mathrm{mm}^{3}$ by the end of the observation period (30 days; mean size, $686 \pm 107 \mathrm{~mm}^{3}$ at the end of observation). By contrast, tumors appeared at the injection sites of the mice treated with Ad-shGTSF1-transfected (GTSF1-negative control) HepG2 cells, and grew from $10-450 \mathrm{~mm}^{3}$ by the end of the observation period (30 days; mean size, $448 \pm 92 \mathrm{~mm}^{3}$ at the end of observation). A total of 30 days following injection, the sizes of the tumors produced in the flanks of mice injected with GTSF1 was increased compared with in those mice treated with Ad-shGTSF1 (GTSF1-negative control) $(\mathrm{P}<0.05)$. Consistently, the tumor weight in mice following injection with Ad-shNC-transfected (GTSF1-positive) HepG2 cells grew from $430-810 \mu \mathrm{g}$ (mean weight, $610 \pm 98 \mu \mathrm{g}$ by the end of observation), whereas mice treated with the Ad-shGTSF1-transfected (GTSF1-negative control) HepG2 cells exhibited tumors weighing 190-450 $\mu \mathrm{g}$ (mean weight, 308 $\pm 73 \mu \mathrm{g}$ by the end of observation) (Fig. 2B; $\mathrm{P}<0.05)$. There were significant 
A

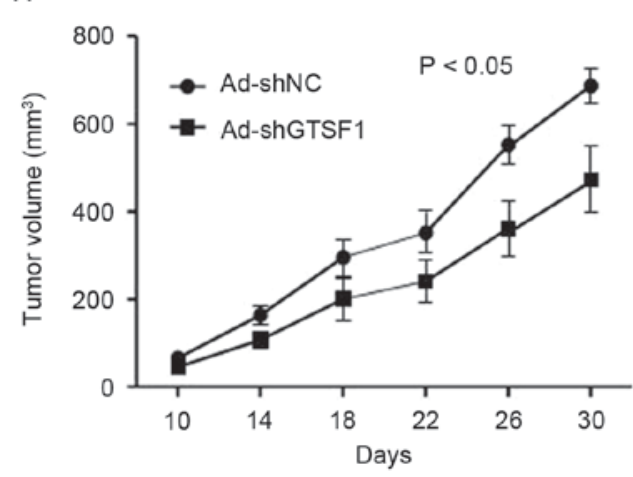

B

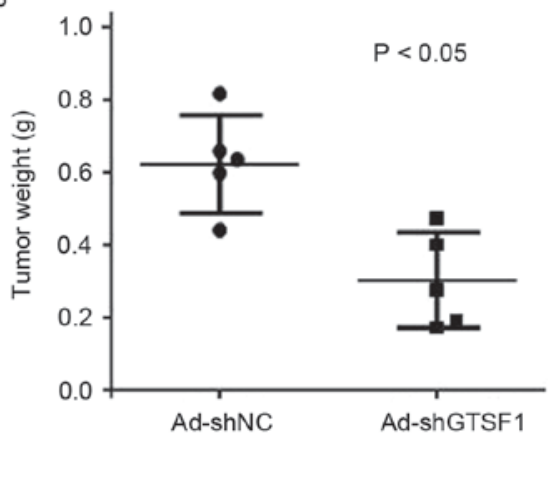

Figure 2. Effect of GTSF1 on tumor cell growth in vivo. (A) Effect of GTSF1 on tumor volume in a nude mouse xenograft model. GTSF1- and Non-GTSF1-transfected HepG2 cells $\left(5 \times 10^{5}\right.$ or $1 \times 10^{6}$, respectively) were injected subcutaneously into either posterior flank of the nude mice. (B) Effect of GTSF1 on tumor weight in nude mice. Images illustrate the representative features of tumor growth 5-6 weeks following inoculation. Ad-shGTSF1 (GTSF1-negative), negative control; GTSF1, gametocyte specific factor 1 gene; Ad-shNC (GTSF1-positive).

differences in the sizes and weights of tumors between those mice injected with GTSF1 and those without it, as determined by a Student's t-test.

Expression of GTSF1 mRNA is a prerequisite for proliferation in hepatoma cell lines. In order to investigate the function of GTSF1 mRNA, two siRNA (GTSF1-siRNA1 and GTSF1-siRNA2) targeting GTSF1 were designed to analyze the mRNA expression levels in PLC/PRF/5 and Huh-7 cell lines. As an initial step in this protocol, the GTSF1 mRNA levels in PLC/PRF/5 and Huh-7 cells were examined, subsequent to transfection with siRNA1-GTSF1, siRNA2-GTSF1 and GTSF1 mRNA (si-NC). Using qPCR, reduced levels of GTSF1 mRNA were identified in human liver cancer cells in the two groups with siRNA targeting GTSF1, compared with the GTSF1 mRNA (si-NC). As indicated in Fig. 3A and B, a 7 day-long regimen of GTSF1 mRNA si-NC transfection was used to achieve a sustained increased level of GTSF1 mRNA expression in the PLC/PRF/5 and Huh-7 cell lines, without two siRNAs, and analyzed using a Student's t-test $(\mathrm{P}<0.01)$. To investigate the potential role of GTSF1 in tumor proliferation, colony formation assays were used to measure the proliferation of $\mathrm{PLC} / \mathrm{PRF} / 5$ and Huh-7 cells transfected with siRNA1-GTSF1 in duplicate, siRNA2-GTSF1 in duplicate, GTSF1 mRNA (si-NC) or with no transfection. Notably, siRNA1-GTSF1 or siRNA2- GTSF1-transfected cells exhibited fewer and smaller colonies compared with GTSF1 mRNA-transfected and non-transfected cells from days 4-6, as determined using a Student's t-test $(\mathrm{P}<0.05)$, as demonstrated in Fig. 3C and D. This result suggests that the GTSF1 gene serves an important role in the proliferation of liver cancer cells. Notably, these results were confirmed by one-way ANOVA followed by an S-N-K post-hoc test. Consistent with the outcomes of the Student's t-tests, there were significant differences between GTSF1 mRNA expression levels and cell proliferation when comparing the GTSF1-transfected cells with the siRNA1 or siRNA2- GTSF1-transfected cells $(\mathrm{P}<0.05)$; no significant difference was present between the siRNA1- and the siRNA2-GTSF1-transfected cells $(\mathrm{P}>0.05)$, as evaluated using ANOVA and S-N-K analysis. These data indicate a growth-proliferation role for GTSF1 mRNA expression, and suggest that it is a prerequisite for the proliferation of hepatoma cell lines.

\section{Discussion}

Hepatocarcinogenesis is a complex and multistep process that involves the accumulation of genetic and epigenetic alterations in regulatory genes. The identification of cancer-associated molecules may lead to the development of novel molecular targets for treatment, and of biomarkers for predicting prognosis (11). The various etiological factors of HCC, including $\mathrm{HBV}$ infection and hepatitis $\mathrm{C}$ virus infection, may affect different signaling pathways (12). Therefore, multiple genetic and epigenetic factors may affect HCC development (13). It has previously been reported in several studies that the GTSF1 gene participates in DNA methylation and retrotransposon activation in germ cells, particularly in cell proliferation, and that it is present in MF tumor samples, suggesting that it may serve a role in the apoptosis resistance of $\mathrm{MF}(7,9,14)$. Therefore, it may be concluded that the GTSF1 gene may serve a role as a proliferation factor during various physiological processes, including growth, development, differentiation and reproduction in animals and plants. In terms of liver cancer, there have been numerous studies regarding the molecular markers associated with the development of liver cancer, and gene signatures with diagnostic and prognostic potential have been identified by the gene expression profiling of tumor tissues (15-17). In the present study, it was identified that GTSF1 mRNA expression in liver cancer tissue samples was increased compared with its expression in adjacent non-tumor liver tissues and healthy control liver tissues. Due to a statistically significant difference between the patients with liver cancer and the healthy controls $(\mathrm{P}<0.05)$, GTSF1 expression levels may be a potential biomarker for liver cancer diagnosis. AFP is a glycoprotein known to be expressed in HCC and is secreted into the blood of $\sim 70 \%$ of patients with liver cancer. Therefore, serum AFP levels are useful for the early detection and differential diagnosis of HCC (18). For patients who undergo curative hepatectomy for localized HCC, AFP levels are also useful for the detection of recurrence, and are associated with prognosis (19-21). Based on the data of the present study, GTSF1 mRNA was expressed in $87.5 \%$ of AFP-positive samples, but only in 50\% of AFP-negative samples. Although there was no statistically significant difference between these two groups ( $\chi^{2}$ test, $\left.\mathrm{P}=0.129\right)$, the proportion of samples that 
A

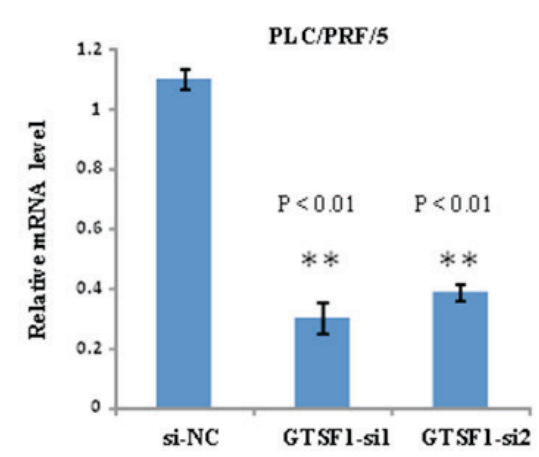

B

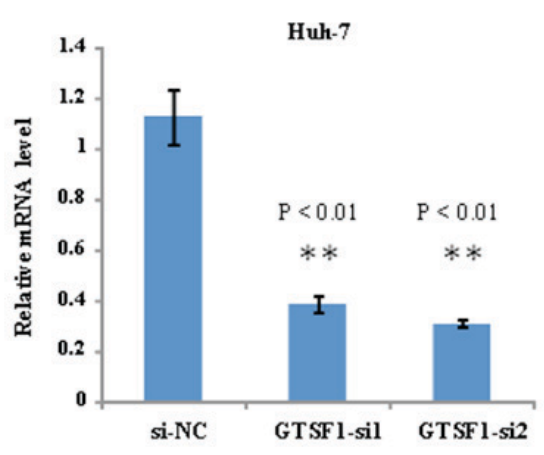

C

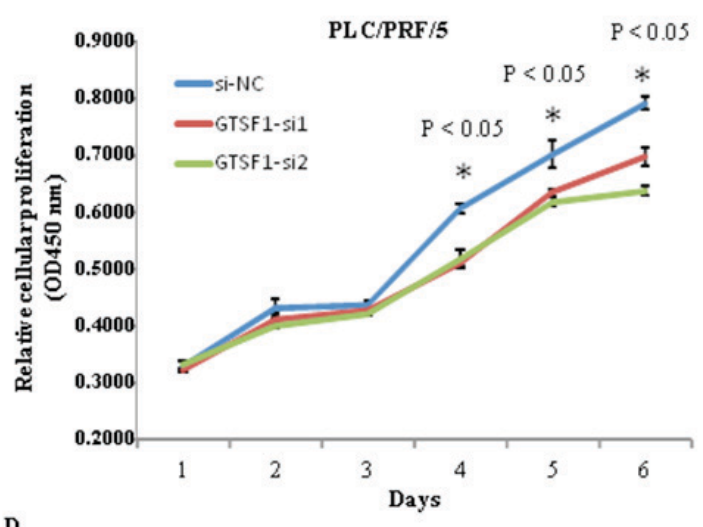

D

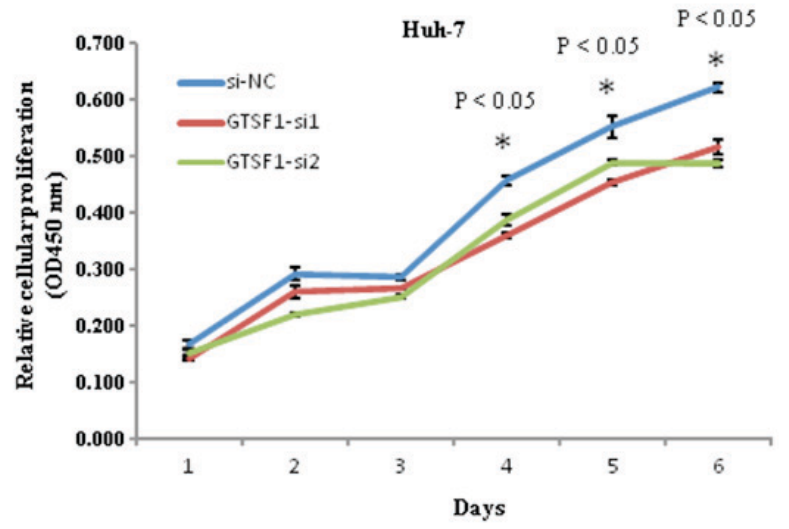

Figure 3. Analysis of GTSF1 mRNA expression levels in human liver cancer cell lines following transfection with two siRNA molecules targeting GTSF1. (A) Silencing of relative GTSF1 mRNA expression in PLC/PRF/5 cell lines using siRNA1 and siRNA2. Error bars denote the standard deviation, and calculated ${ }^{* *} \mathrm{P}<0.01$ compared with siNC. (B) Silencing of relative GTSF1 mRNA expression in Huh-7 cell lines using siRNA1 and siRNA2. (C) The relative cellular proliferation level of GTSF1-siNC-, GTSF1-siRNA1- and GTSF1-siRNA2-transfected PLC/PRF/5 cells from days 1-6. The level of relative proliferation was measured at $450 \mathrm{~nm}$ absorbance after $1 \mathrm{~h}$ of incubation. The relative proliferation of PLC/PRF/5 cells was significantly decreased from days 3-6, when compared with the GTSF1-siNC and siRNA groups. Cell growth assays were performed in 96-well microtiter plates and all assays were performed in triplicate. GTSF1 expression without siRNA interference (GTSF1-siNC) was used as the positive control. (D) The relative cellular proliferation level of Huh-7 cells following GTSF1-siNC, GTSF1-siRNA1 and GTSF1-siRNA2 interference. ${ }^{* *} \mathrm{P}<0.01$ (relative GTSF1 mRNA expression in GTSF1-siNC groups compared with GTSF1-siRNA1 groups and in GTSF1-siNC groups compared with GTSF1-siRNA2 groups). "P<0.05 (relative proliferation in GTSF1-siRNA1 and GTSF1-siRNA2 groups compared with the GTSF1-siNC group). NC, negative control; GTSF1, gametocyte specific factor 1; si, small interfering.

were GTSF1-positive was almost equivalent to the number that were AFP-positive. Notably, GTSF1 expression was identified in AFP-negative liver cancer tissue samples, and may be a potential biomarker for diagnosis in patients with AFP-negative liver cancer. In addition, there was no statistical significance between GTSF1 expression in the non-HBV-infected liver cancer samples and the HBV-infected liver cancer specimens observed. It may be hypothesized that GTSF1 expression in liver cancer samples is irrelevant to HBV infection. Notably, GTSF1 expression was significantly upregulated in the majority of liver cancer tissues examined, but was not expressed in the adjacent non-tumor normal liver tissue. These results suggest that increased GTSF1 expression is a frequent event in human liver cancer tissues and may be involved in hepatocarcinogenesis.

During tumor progression, the number, type, distribution and expression levels of tumor markers in patients with liver cancer exhibit variations that are closely associated with the occurrence, development, metastasis, treatment response and prognosis of tumors and patients (22). Few data are available concerning the molecular mechanisms by which mRNAs modulate the process of tumorigenesis and the behavior of cancer cells. To explore the roles of GTSF1 in the liver in vivo, transfected HepG2 cells were generated and injected to a mouse model using a clone of a constructed GTSF1 gene plasmid. In agreement with previous observations that GTSF1 was frequently upregulated in hepatoma cell lines, and that GTSF1 may increase colony formation in vitro, it was demonstrated that GTSF1 promoted tumor growth in vivo. All these data emphasize a fundamental role of GTSF1 in tumorigenesis, particularly in the development of liver cancer. The present study revealed that GTSF1 significantly increased tumorigenicity in Ad-shNC-transfected (GTSF1-positive) HepG2 cells in a nude mouse xenograft model, whereas the absence of GTSF1 inhibited increases in the size and weight of the tumors. Therefore, GTSF1 expression may serve a critical role in the proliferation of HCC tumor cells in vivo. Notably, the HepG2 cell line, originally thought to be an HCC cell line, was identified as a hepatoblastoma cell line (23). This is important, as differences exist between HepG2 cells and native human hepatocytes, including in the drug-processing proteins in liver tissues and the genetic changes that occur in HCC; 
these changes can affect protein concentrations and the copy number (24-26). Conversely, genetic changes involved in the development of liver cancer, the cancer protein secretomes and the biological significance of human leucocyte antigen expression in HCC tumors closely resemble those of the HepG2 cells observed in previous studies (27-29).

Compared with previous studies, the results of the present study indicated that GTSF1-transfected HepG2 cells injected into mice produced tumors larger in size (mean, $686 \pm 107 \mathrm{~mm}^{3}$ ) and weight (mean, 610 $\pm 98 \mu \mathrm{g}$ ) compared with the tumors observed in the mice injected with GTSF1-negative HepG2 cells (mean size, $448 \pm 92 \mathrm{~mm}^{3}$; mean weight, $308 \pm 73 \mu \mathrm{g}$; Fig. 2A and B). Therefore, the tumors were bigger and heavier in the model that used GTSF1-transfected HepG2 cells, compared with in those cells transfected with the negative control, which confirmed that the introduction of GTSF1 significantly increases tumorigenicity in vivo. Although the misidentified hepatoblastoma HepG2 cell line was used in the present study, this is unlikely to have affected the ability of GTSF1 to initiate tumor growth in vivo when using HepG2 cells as the vector. Due to the limitations of using the HepG2 cell line, these results may indicate that GTSF1 significantly promoted in vivo tumorigenicity in malignant liver tumors in general, rather than in HCC specifically. The mechanism for this apparent GTSF1 gene proliferation function in $\mathrm{HCC}$ in vivo should be elucidated using verified HCC cell lines in the future.

To validate the effects of the GTSF1 gene on cell proliferation and growth, siRNA protocols were used in the present study. As it is possible to generate siRNAs and miRNAs targeted against any cellular RNA, these molecules may be used to downregulate the expression of almost any disease-causing gene (30-32). The in vitro data obtained during the present study indicated that GTSF1 knockdown by siRNA1 or siRNA2 significantly reduced the proliferation of PLC/PRF/5 and Huh-7 cells. The cell proliferation, as evaluated by colony formation, of liver cancer cell lines without GTSF1 siRNA was significantly decreased in HCC cells. In addition, the levels of GTSF1 expression were significantly decreased following GTSF1 knockdown in PLC/PRF/5 and Huh-7 cells. Therefore, it was hypothesized that the result of interfering with the expression of GTSF1 in PLC and HepG2 cells may be due to GTSF1 overexpression in the tissues of patients with liver cancer, and that this result may be associated with the observations concerning HCC tumor size and weight from the mouse model. These results suggest that the overexpression of GTSF1 is involved in regulating liver cancer proliferation. The use of siRNA/miRNA is considered to have great therapeutic potential, as it is possible to generate siRNA/miRNA-based silencing of any gene implicated in HCC (33). Notably, GTSF1 mRNA expression was significantly upregulated in the majority of the cancer cell lines and cancer tissues examined, and that GTSF1 not only increased colony formation in vitro but also tumorigenicity in vivo.

In summary, the present study revealed the GTSF1 mRNA expression profile in liver cancer, and examined the potential role of GTSF1 in tumorigenesis. The data suggest an important role for the GTSF1 gene in the molecular etiology of hepatocarcinogenesis, and suggest the potential application of GTSF1 mRNA expression in liver cancer diagnosis and therapy. Due to the limitations associated with the small number of cases involved, the absence of data on hepatoblastoma tissues derived from patients, and the patient cohort all originated from a single region in the present study, the function of GTSF1 mRNA and protein expression in liver cancer requires additional study.

\section{Acknowledgements}

The present study was supported by the Science of Shanghai Songjiang District Fund in China (grant no. 13SJGGYY28), and by the Key project of Shanghai Songjiang District Planning and Growth Committee (grant no. 2012-III).

\section{References}

1. Balogh J, Victor D III, Asham EH, Burroughs SG, Boktour M, Saharia A, Li X, Ghobrial RM and Monsour HP Jr: Hepatocellular carcinoma: A review. J Hepatocell Carcinoma 3: 41-53, 2016.

2. Torre LA, Bray F, Siegel RL, Ferlay J, Lortet-Tieulent J and Jemal A: Global cancer statistics, 2012. CA Cancer J Clin. 65: 87-108. 2015

3. Caldwell S and Park SH: The epidemiology of hepatocellular cancer: From the perspectives of public health problem to tumor biology. J Gastroenterol 44 (Suppl 19): S96-S101, 2009.

4. Ferenci P, Fried M, Labrecque D, Bruix J, Sherman M, Omata M, Heathcote J, Piratsivuth T, Kew M, Otegbayo JA, et al: Hepatocellular carcinoma (HCC): A global perspective. J Clin Gastroenterol 44: 239-245, 2010.

5. Tsuchiya N, Sawada Y, Endo I, Saito K, Uemura Y and Nakatsura T: Biomarkers for the early diagnosis of hepatocellular carcinoma. World J Gastroenterol 21: 10573-10583, 2015.

6. Zucman-Rossi J, Villanueva A, Nault JC and Llovet JM: Genetic landscape and biomarkers of hepatocellular carcinoma. Gastroenterology 149: 1226-1239.e4, 2015.

7. Yoshimura T, Toyoda S, Kuramochi-Miyagawa S, Miyazaki T, Miyazaki S, Tashiro F, Yamato E, Nakano T and Miyazaki J: Gtsf1/Cue110, a gene encoding a protein with two copies of a CHHC $\mathrm{Zn}$-finger motif, is involved in spermatogenesis and retrotransposon suppression in murine testes. Dev Biol 335: 216-227, 2009.

8. Krotz SP, Ballow DJ, Choi Y and Rajkovic A: Expression and localization of the novel and highly conserved gametocyte-specific factor 1 during oogenesis and spermatogenesis. Fertil Steril 91 (5 Suppl): S2020-S2024, 2009.

9. van Kester MS, Borg MK, Zoutman WH, Out-Luiting JJ, Jansen PM, Dreef EJ, Vermeer MH, van Doorn R, Willemze R and Tensen CP: A meta-analysis of gene expression data identifies a molecular signature characteristic for tumor-stage mycosis fungoides. J Invest Dermatol 132: 2050-2059, 2012.

10. Livak KJ and Schmittgen TD: Analysis of relative gene expression data using real-time quantitative PCR and the 2(-Delta Delta C(T)) method. Methods 25: 402-408, 2001.

11. Shimizu D, Inokawa Y, Sonohara F, Inaoka K and Nomoto S: Search for useful biomarkers in hepatocellular carcinoma, tumor factors and background liver factors (Review). Oncol Rep 37: 2527-2542, 2017.

12. Shao YY, Hsu CH and Cheng AL: Predictive biomarkers of antiangiogenic therapy for advanced hepatocellular carcinoma: Where are we? Liver Cancer 2: 93-107, 2013.

13. Llovet JM and Bruix J: Molecular targeted therapies in hepatocellular carcinoma. Hepatology 48: 1312-1327, 2008.

14. Yoshimura T, Miyazaki T, Toyoda S, Miyazaki S, Tashiro F, Yamato E and Miyazaki J: Gene expression pattern of Cue110: A member of the uncharacterized UPF0224 gene family preferentially expressed in germ cells. Gene Expr Patterns 8: 27-35, 2007.

15. Tanabe KK, Lemoine A, Finkelstein DM, Kawasaki H, Fujii T, Chung RT, Lauwers GY, Kulu Y, Muzikansky A, Kuruppu D, et al: Epidermal growth factor gene functional polymorphism and the risk of hepatocellular carcinoma in patients with cirrhosis. JAMA 299: 53-60, 2008.

16. Li M, Zhao H, Zhang X, Wood LD, Anders RA, Choti MA, Pawlik TM, Daniel HD, Kannangai R, Offerhaus GJ, et al: Inactivating mutations of the chromatin remodeling gene ARID2 in hepatocellular carcinoma. Nat Genet 43: 828-829, 2011.

17. Jin F, Xiong WJ, Jing JC, Feng Z, Qu LS and Shen XZ: Evaluation of the association studies of single nucleotide polymorphisms and hepatocellular carcinoma: A systematic review. J Cancer Res Clin Oncol 137: 1095-1104, 2011. 
18. Snowberger N, Chinnakotla S, Lepe RM, Peattie J, Goldstein R, Klintmalm GB and Davis GL: Alpha fetoprotein, ultrasound, computerized tomography and magnetic resonance imaging for detection of hepatocellular carcinoma in patients with advanced cirrhosis. Aliment Pharmacol Ther 26: 1187-1194, 2007.

19. Grat M, Kornasiewicz O, Lewandowski Z, Hołówko W, Grạt K Kobryń K, Patkowski W, Zieniewicz K and Krawczyk M: Combination of morphologic criteria and $\alpha$-fetoprotein in selection of patients with hepatocellular carcinoma for liver transplantation minimizes the problem of posttransplant tumor recurrence. World J Surg 38: 2698-2707, 2014.

20. Raoul JL, Park JW, Kang YK, Finn RS, Kim JS, Yeo W, Polite BN, Chao Y, Walters I, Baudelet $\mathrm{C}$ and Lencioni R: Using modified RECIST and alpha-fetoprotein levels to assess treatment benefit in hepatocellular carcinoma. Liver Cancer 3: 439-450, 2014.

21. Furihata T, Sawada T, Kita J, Iso Y, Kato M, Rokkaku K, Shimoda M and Kubota K: Serum alpha-fetoprotein level per tumor volume reflects prognosis in patients with hepatocellular carcinoma after curative hepatectomy. Hepatogastroenterology 55: 1705-1709, 2008.

22. Zhao YJ, Ju Q and Li GC: Tumor markers for hepatocellular carcinoma. Mol Clin Oncol 1: 593-598, 2013.

23. López-Terrada D, Cheung SW, Finegold MJ and Knowles BB: Hep G2 is a hepatoblastoma-derived cell line. Hum Pathol 40 $1512-1515,2009$

24. Hart SN,Li Y, Nakamoto K, Subileau EA, Steen D and Zhong XB: A comparison of whole genome gene expression profiles of HepaRG cells and HepG2 cells to primary human hepatocytes and human liver tissues. Drug Metab Dispos 38: 988-994, 2010.

25. Wiśniewski JR, Vildhede A, Norén A and Artursson P: In-depth quantitative analysis and comparison of the human hepatocyte and hepatoma cell line HepG2 proteomes. J Proteomics 136 234-247, 2016
26. Capes-Davis A, Theodosopoulos G, Atkin I, Drexler HG, Kohara A, MacLeod RA, Masters JR, Nakamura Y, Reid YA, Reddel RR and Freshney RI: Check your cultures! A list of cross-contaminated or misidentified cell lines. Int J Cancer 127: $1-8,2010$.

27. Cevik D, Yildiz G and Ozturk M: Common telomerase reverse transcriptase promoter mutations in hepatocellular carcinomas from different geographical locations. World J Gastroenterol 21: 311-317, 2015

28. Srisomsap C, Sawangareetrakul P, Subhasitanont $P$, Chokchaichamnankit D, Chiablaem K, Bhudhisawasdi V, Wongkham S and Svasti J: Proteomic studies of cholangiocarcinoma and hepatocellular carcinoma cell secretomes. J Biomed Biotechnol 2010: 437143, 2010.

29. Wadee AA, Paterson A, Coplan KA and Reddy SG: HLA expression in hepatocellular carcinoma cell lines. Clin Exp Immunol 97: 328-333, 1994.

30. Dapas B, Farra R, Grassi M, Giansante C, Fiotti N, Uxa L, Rainaldi G, Mercatanti A, Colombatti A, Spessotto P, et al: Role of E2F1-cyclin E1-cyclin E2 circuit in human coronary smooth muscle cell proliferation and therapeutic potential of its downregulation by siRNAs. Mol Med 15: 297-306, 2009.

31. Farra R, Dapas B, Pozzato G, Giansante C, Heidenreich O, Uxa L, Zennaro C, Guarnieri G and Grassi G: Serum response factor depletion affects the proliferation of the hepatocellular carcinoma cells HepG2 and JHH6. Biochimie 92: 455-463, 2010.

32. Spänkuch B and Strebhardt K: Combinatorial application of nucleic acid-based agents targeting protein kinases for cancer treatment. Curr Pharm Des 14: 1098-1112, 2008.

33. Farra R, Grassi M, Grassi G and Dapas B: Therapeutic potential of small interfering RNAs/micro interfering RNA in hepatocellular carcinoma. World J Gastroenterol 21: 8994-9001, 2015. 